\title{
Aptamer-targeted inhibition of mTOR in T cells enhances antitumor immunity
}

\author{
Alexey Berezhnoy, ${ }^{1}$ Iris Castro, ${ }^{2}$ Agata Levay, ${ }^{1}$ Thomas R. Malek, ${ }^{2}$ and Eli Gilboa ${ }^{1}$ \\ 1Dodson Interdisciplinary Immunotherapy Institute, Sylvester Comprehensive Cancer Center, and 2Department of Microbiology and Immunology, \\ Miller School of Medicine, University of Miami, Miami, Florida, USA.
}

\begin{abstract}
Recent studies have underscored the importance of memory $\mathrm{T}$ cells in mediating protective immunity against pathogens and cancer. Pharmacological inhibition of regulators that mediate $T$ cell differentiation promotes the differentiation of activated $\mathrm{CD8}^{+} \mathrm{T}$ cells into memory cells. Nonetheless, pharmacological agents have broad targets and can induce undesirable immunosuppressive effects. Here, we tested the hypothesis that aptamer-targeted siRNA inhibition of mTOR complex 1 (mTORC1) function in $\mathrm{CD8}^{+} \mathrm{T}$ cells can enhance their differentiation into memory $T$ cells and potentiate antitumor immunity more effectively than the pharmacologic inhibitor rapamycin. To specifically target activated cells, we conjugated an siRNA targeting the mTORC1 component raptor to an aptamer that binds 4-1BB, a costimulatory molecule that is expressed on $\mathrm{CD8}^{+} \mathrm{T}$ cells following TCR stimulation. We found that systemic administration of the 4-1BB aptamer-raptor siRNA to mice downregulated mTORC1 activity in the majority of $\mathrm{CD8}^{+} \mathrm{T}$ cells, leading to the generation of a potent memory response that exhibited cytotoxic effector functions and enhanced vaccine-induced protective immunity in tumor-bearing mice. In contrast, while treatment with the general mTORC1 inhibitor rapamycin also enhanced antigen-activated $\mathrm{CD8}^{+} \mathrm{T}$ cell persistence, the cytotoxic effector functions of the reactivated memory cells were reduced and the alloreactivity of DCs was diminished. Consistent with the immunological findings, mice treated with rapamycin, but not with 4-1BB aptamer-raptor siRNA, failed to reject a subsequent tumor challenge.
\end{abstract}

\section{Introduction}

Studies in mice have highlighted the importance of persistence of the vaccine-induced immune response (immunological memory) in mediating protective immunity against infectious diseases and cancer (1). A correlation between $\mathrm{T}$ cell memory and protective immunity was also seen in nonhuman primates vaccinated against SIV (2-4) and in cancer patients treated with adoptively transferred T cells (5-9). Vaccination protocols must therefore be designed for or complemented with treatments that promote the generation of strong and long-lasting memory responses.

Multiple extrinsic pathways control memory differentiation by regulating the balanced expression of intracellular mediators in activated $\mathrm{CD}^{+} \mathrm{T}$ cells (10-13). For example, elevated levels of mTOR (14), T-bet (15), BLIMP1 (16-18), or GSK3 (19) promote the accumulation of short-lived effectors, whereas products like BCL6 (18), Eomes $(20,21)$, TRAF6 $(22)$, or TCF1 $(21,23)$ promote the accumulation of memory cells. Notably, inhibiting the mediators of effector differentiation using genetic means or, whenever available, pharmacological agents, redirected the activated $\mathrm{T}$ cells to differentiate along the memory pathway. For example, pharmacological inhibition of mTOR with rapamycin in lymphocytic choriomeningitis virusinfected (LCMV-infected) mice led to enhanced differentiation of the LCMV-specific CD8 ${ }^{+} \mathrm{T}$ cells into memory cells (14); activation of AMPK with metformin promoted the development of memory $\mathrm{CD}^{+} \mathrm{T}$ cells (22); and inhibition of GSK3 with TWS119 led to the differentiation of memory precursors with self-renewal capacity (19). The therapeutic potential of promoting memory responses with pharmacological agents like rapamycin, TWS119, or metformin was demonstrated in murine studies using antigen-specific transgenic

Conflict of interest: The authors have declared that no conflict of interest exists. Citation for this article: J Clin Invest. 2014;124(1):188-197. doi:10.1172/JCI69856
$\mathrm{CD}^{+} \mathrm{T}$ cells adoptively transferred to tumor-bearing mice $(19,24)$ or to mice subsequently challenged with tumor or recombinant virus $(14,22,25)$. With one exception (19), the transgenic T cells targeted a potent model antigen, chicken OVA, ectopically expressed in the tumor or virus. While providing a proof-of-concept that the promotion of memory responses with pharmacological agents can enhance protective immunity, the question remains whether such strategies will be therapeutically useful in clinical settings.

Pharmacological agents, given the broad distribution of their targets, can also exhibit undesirable immune (suppressive) and nonimmune effects, raising significant, if not insurmountable, challenges in translating those finding to human patients. For example, rapamycin inhibition of mTOR promotes the development of immunosuppressive regulatory Foxp $3^{+} \mathrm{CD} 4^{+} \mathrm{T}$ cells (Tregs) (26), polarizes DCs to become tolerogenic APCs $(27,28)$, and prevents the tissue trafficking of activated T cells (ref. 29 and reviewed in refs. 30,31). In addition, the development of pharmacological agents designed to modulate the function of intracellular targets that are not accessible to antibodies ("undruggable targets") is challenging, and their availability, especially for clinical use, is limited. There are currently no pharmacological agents available for the inhibition of intracellular mediators such as BLIMP1 or T-bet, however, their inhibition could provide substantial advantages in promoting immunological memory.

In this study, we describe a versatile, broadly applicable, and clinically feasible approach to promoting the generation of memory T cell responses that addresses the main limitations of pharmacological agents. We used siRNAs to downregulate intracellular mediators of $\mathrm{CD}^{+} \mathrm{T}$ cell effector differentiation. RNAi is broadly applicable to virtually any target, including "nondruggable" intracellular targets such as BLIMP1 or T-bet. To reduce the undesirable effects that could result from the downregulation of the siRNA targets in other cell types, we 
A
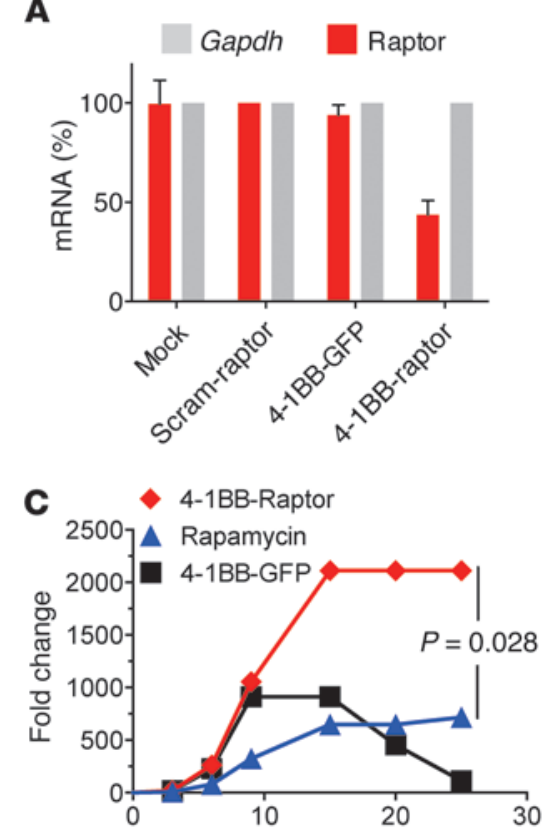

B
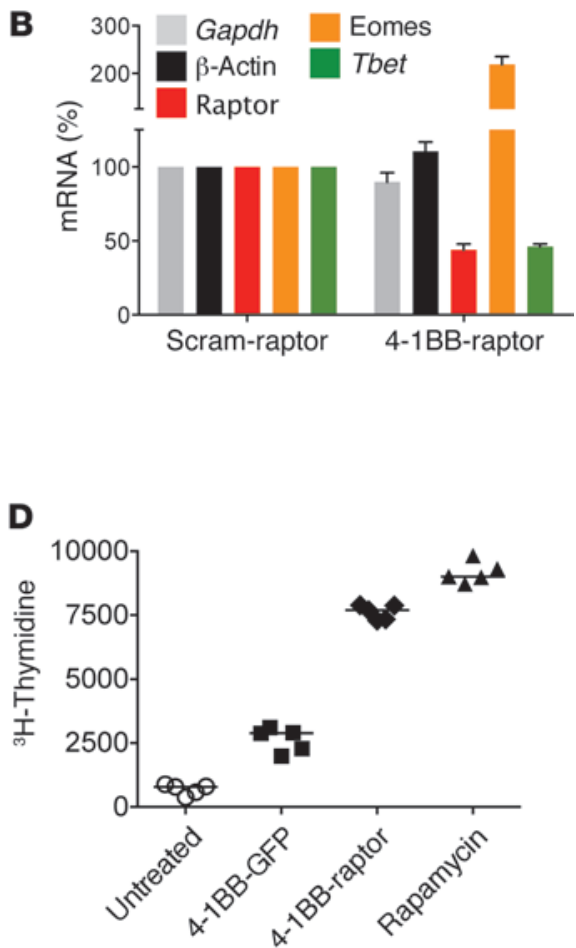

\section{Figure 1}

4-1BB-raptor conjugate downregulates raptor in cultured OT-I cells and promotes their persistence. (A and B) Splenocytes from OT-I mice were activated with OVA peptides and incubated with scrambled aptamer-raptor siRNA conjugate (Scram raptor), 4-1BB aptamer-GFP siRNA conjugate (4-1BB-GFP), or 4-1BB aptamer-raptor siRNA conjugate (4-1BB-raptor) three times every 8 hours at a concentration of $0.8 \mu \mathrm{M}$. Forty-eight hours after the last treatment, cells were harvested, and mRNA was quantified by qRT-PCR. $n=4(\mathbf{A}) ; n=2$ (B). (C and D) Splenocytes from OT-I mice were activated with OVA peptide and incubated with rapamycin $(75 \mathrm{ng} / \mathrm{ml})$ three times daily or with 4-1BB-GFP and 4-1BB-raptor conjugates $(0.8 \mu \mathrm{M}$, three times every 8 hours). After the last treatment, $10^{6}$ cells were transferred to $5 \mathrm{ml}$ of fresh media supplemented with IL-7 $(5 \mathrm{ng} / \mathrm{ml})$ and cultured for 3 to 4 weeks. Every 3 to 5 days, viable cells were counted, and $10^{6}$ cells were passed to a fresh culture. (C) Viable cells $(n=5)$. (D) On day 25, cells were counted and plated at $10^{4}$ cells per well in a 96-well plate in quintuplicate. Cells were stimulated overnight with $0.01 \mathrm{nM}$ of OVA. ${ }^{3 \mathrm{H}}$-thymidine $(1 \mu \mathrm{Ci})$ was added to each well 6 hours prior to harvesting and counting. $P<0.001$ among all groups $(n=3)$.

targeted systemically administered siRNA to $\mathrm{CD}^{+} \mathrm{T}$ cells using oligonucleotide aptamers. Aptamers are high-affinity, single-stranded nucleic acid ligands that can be isolated using a combinatorial chemistry process known as SELEX (systematic evolution of ligands by exponential enrichment) (32). Aptamers exhibit specificity and avidity comparable to or exceeding those of antibodies and can be generated against most targets. Recent publications have illustrated the feasibility and therapeutic potential of aptamers as targeting ligands to eradicate tumors (33), sensitize tumor cells to radiation therapy (34), inhibit HIV replication $(35,36)$, and potentiate tumor immunity $(37$, 38). In this study, we show that aptamer-targeted siRNA inhibition of $\mathrm{mTOR}$ function in $\mathrm{CD}^{+} \mathrm{T}$ cells potentiates a vaccine-induced memory and an antitumor immunity that are quantitatively comparable, but qualitatively superior, to rapamycin treatment.

\section{Results}

Aptamer-targeted siRNA knockdown of raptor RNA and inbibition of $m$ TORC1 activity. To test whether $\mathrm{CD}^{+} \mathrm{T}$ cell memory can be enhanced by targeted inhibition of $\mathrm{mTOR}$, we conjugated an
siRNA specific to raptor, a component of the mammalian target of rapamycin complex 1 (mTORC1) (30), to an aptamer that binds to 4-1BB (39), a major costimulatory molecule that is transiently expressed on $\mathrm{CD}^{+} \mathrm{T}$ cells following TCR stimulation $(40,41)$. Incubation of activated OT-I cells, transgenic $\mathrm{CD}^{+} \mathrm{T}$ cells that recognize a determinant of the chicken OVA product (42), with 4-1BB aptamer-raptor siRNA (4-1BB-raptor) conjugate downregulated raptor mRNA in an aptamer-dependent manner (Figure 1A). Suggestive evidence that the observed reduction in raptor mRNA levels led to the inhibition of mTOR activity was provided by our observations that raptor mRNA downregulation was accompanied by downregulation of Tbet mRNA and upregulation of Eomes mRNA (refs. 25, 43, and Figure 1B) and that the 4-1BB-raptor conjugate-treated OT-I cells exhibited prolonged survival and enhanced proliferative capacity comparable with that of rapamycin-treated cells as was previously shown (refs. 14, 25, and Figure 1, C and D).

Supplemental Figure 1A (supplemental material available online with this article; doi:10.1172/JCI69856DS1) shows that i.v. administered ${ }^{32} \mathrm{P}-$ labeled 4-1BB, but not scrambled, aptamer-raptor siRNA conjugates become associated with 4-1BBexpressing cells, but not with 4-1BB-negative cells from lymph nodes, bone marrow, and spleen. The systemically administered 4-1BB-raptor conjugate accumulated preferentially in the bone marrow where 4-1BBexpressing memory $\mathrm{CD}^{+} \mathrm{T}$ cells reside, whereas no significant accumulation was noted in the lungs, kidney, or liver, as is often seen upon administration of unformulated siRNA (Supplemental Figure 1B).

To test whether systemic administration of 4-1BB-raptor conjugates is capable of inhibiting mTOR activity in activated $\mathrm{CD}^{+}$ $\mathrm{T}$ cells in vivo, we transferred OT-I cells into C57BL/6 mice, and 2 days later we vaccinated the mice with OVA peptide and treated them with aptamer-siRNA conjugates or with rapamycin. In this experimental system, 4-1BB is upregulated on the OT-I cells as early as 6 hours later, peaks between 12 and 24 hours, and returns to background levels after 48 hours (data not shown). Two days later, we determined conjugate or rapamycin treatment mTORC1 activity in the host cells and donor OT-I cells by measuring the phosphorylation of $\mathrm{S} 6$ ribosomal protein, one of the downstream targets of mTORC1. As shown in Figure 2A, treatment of the OT-I-bearing mice with rapamycin or with 4-1BB-raptor, but not with 4-1BB-GFP, conjugate downregulated mTORC1 activity in $90 \%$ and $59 \%$ of the OT-I cells, respectively. The cell and target specificity of raptor siRNA inhibition is indicated by the fact that rapamycin, but not the 4-1BB-raptor conjugate, also inhibited mTOR activity in a proportion of host cells (Figure $2 \mathrm{~A}$ ) and that the 4-1BB-raptor conjugate inhibited the activity of mTORC1, 
A

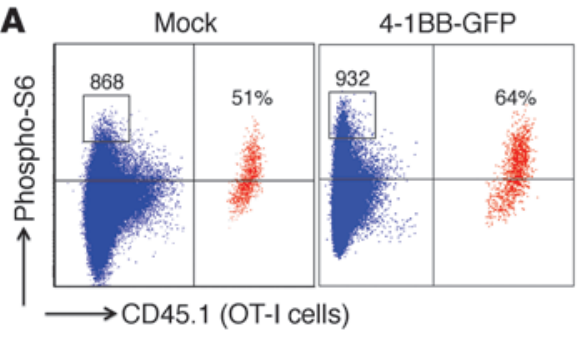

C
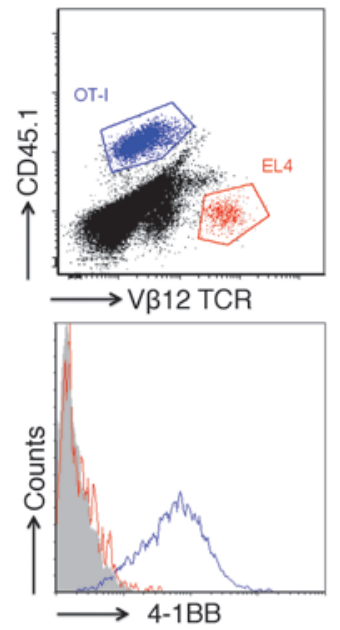

D

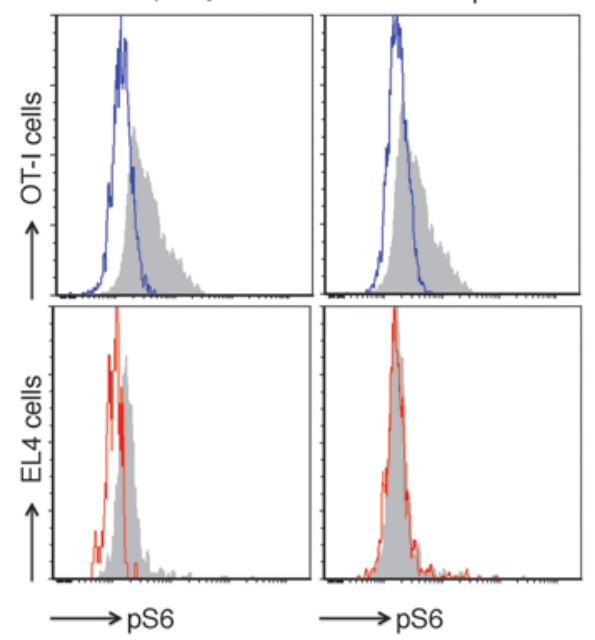

B

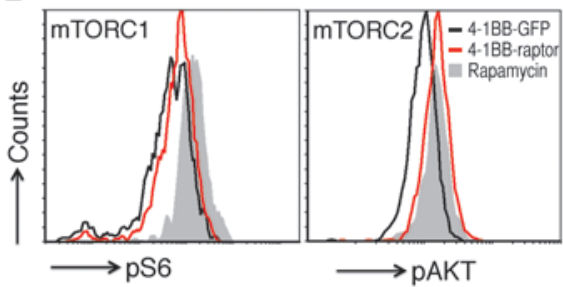

$\mathbf{E}$
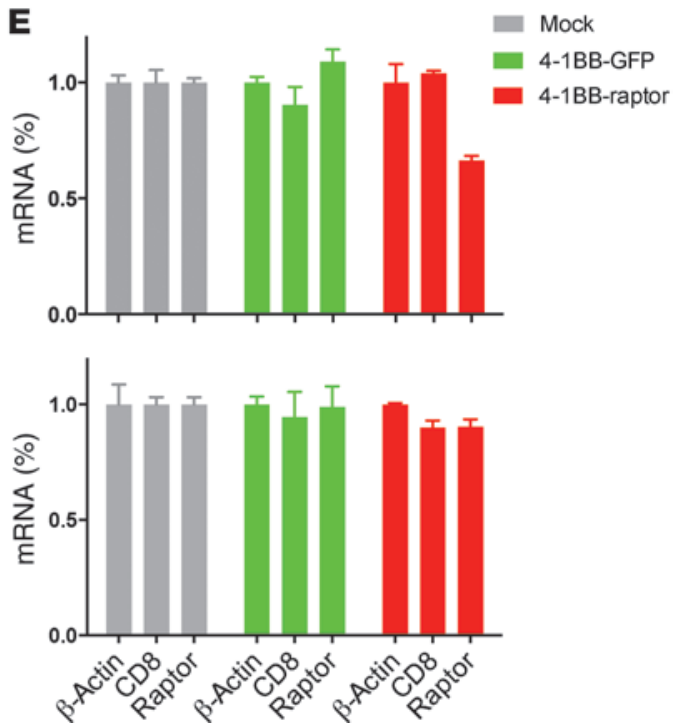

Figure 2

4-1BB-raptor conjugate decreases mTORC1 activity in activated OT-I cells in mice. CD45.2 C57BL/6 mice were transferred with congenic CD45.1 OT-I cells, activated in situ with OVA peptide, and treated either with rapamycin or with 4-1BB aptamer-GFP siRNA (4-1BB-GFP) or 4-1BB aptamer-raptor siRNA (4-1BB-raptor) conjugates, as described in Methods. Forty-eight hours after treatment, splenocytes were stained for (A) phosphorylated S6 riboprotein (mTORC1 activity) and CD45.1 (OT-I cells) $(n=6)$ or (C) CD8, CD45.1, and either phosphorylated S6 (p235) or phosphorylated Akt (p473) (mTORC2 activity) $(n=3)$. (C) Mice were adoptively transferred with $1 \times 10^{6}$ OT-I cells and $4 \times 10^{6}$ T cell EL4 lymphoma cells and injected 24 hours later with OVA peptide and LPS. EL4 cells can be distinguished from host cells by staining with a V $\beta 12$ TCR chain-specific antibody. Twenty-four hours after peptide injection, splenocytes were isolated and stained for OT-I (CD45.1), EL4 (V $\beta 12$ ), and 4-1BB. OT-I and EL4 can be readily distinguished by CD45.1 and VB12 staining. (D) Four and 24 hours after peptide administration, mice were injected i.p. with $1.5 \mu \mathrm{g}$ rapamycin or i.v. with $1 \mathrm{nmole}$ 4-1BB-raptor conjugate, or were left untreated (filled gray area). Mice were analyzed for mTORC1 activity by staining for phosphorylated S6 (pS6) in OT-1 (blue) and EL4 (red) cells. (C and D) $n=1$, with three time points, one of which is shown. (E) Forty-eight hours after peptide LPS vaccination, splenocytes were isolated from 4-1BB-GFP conjugate (green), 4-1BB-raptor conjugate (red), or from mock-treated mice (gray). CD45.1 ${ }^{+} \mathrm{CD} 8{ }^{+}$(OT-I, upper panel) and CD45.1-CD8 ${ }^{+}$(host CD8 ${ }^{+} \mathrm{T}$ cells, lower panel) were sorted to $>99.5 \%$ purity, and $\beta$-actin, $C d 8$, and raptor mRNA levels were quantified in each population by qRT-PCR.

but not mTORC2, whereas rapamycin inhibited both $\mathrm{mTOR}$ complexes, as was previously shown (refs. 44, 45, and Figure 2B). OT-I cells in mice treated with 4-1BB-GFP conjugate expressed more mTORC1 (64\%) compared with mock-treated mice (51\%), suggesting that $4-1 \mathrm{BB}$ costimulation affected mTORC1 expression. This difference, however, was not always seen. The small decrease of phospho-S6-expressing host (CD45.1-) cells seen in the 4-1BB-raptor conjugate-treated mice may correspond to a small fraction of non-CD8 ${ }^{+} \mathrm{T}$ cells that also express $4-1 \mathrm{BB}$ (46, $47)$, reflecting the lack of complete specificity of this targeting protocol. We further evaluated the specificity of aptamer-targeted mTORC1 inhibition by cotransferring mice with OT-I cells together with EL-4 T lymphoma cells that can be readily distinguished by flow cytometry (Figure 2C). Unlike OT-I cells, EL4 cells did not upregulate 4-1BB upon peptide and LPS administration (Figure 2C). As shown in Figure 2D, whereas rapamycin treatment inhibited mTORC1 signaling in both OT-I and EL-4 cells, the administration of 4-1BB-raptor conjugate inhibited mTORC1 in 4-1BB-positive OT-I cells, but not in 4-1BB-negative EL4 cells. Figure $2 \mathrm{E}$ shows that on a population level, raptor RNA, but not CD8 or $\beta$-actin RNA, is reduced in OT-I cells (upper panel), but not in host $\mathrm{CD}^{+}$cells (lower panel) isolated from OT-I-bearing mice treated with 4-1BB-raptor, but not 4-1BB-GFP, conjugate. Given that the efficiency of aptamer-targeted siRNA delivery to circulating OT-I cells was about $60 \%$ as measured by functional analysis of mTORC1 (Figure 2A), the extent of raptor downregulation on a per-cell basis was considerably higher. Supplemental Figure 2A shows that 4-1BB-raptor conjugates accumulated preferentially in the transferred OT-I cells compared with host CD4-, CD8-, CD19-, or F4/80-expressing cells, and Supplemental Figure $2 \mathrm{~B}$ shows that this correlates with inhibition of mTORC1 in OT-I, but not in host CD4-, CD8-, CD19-, or F4/80-expressing cells, whereas treatment with rapamycin led to mTORC1 inhibition in all cell types analyzed. Overall, these observations demonstrate 

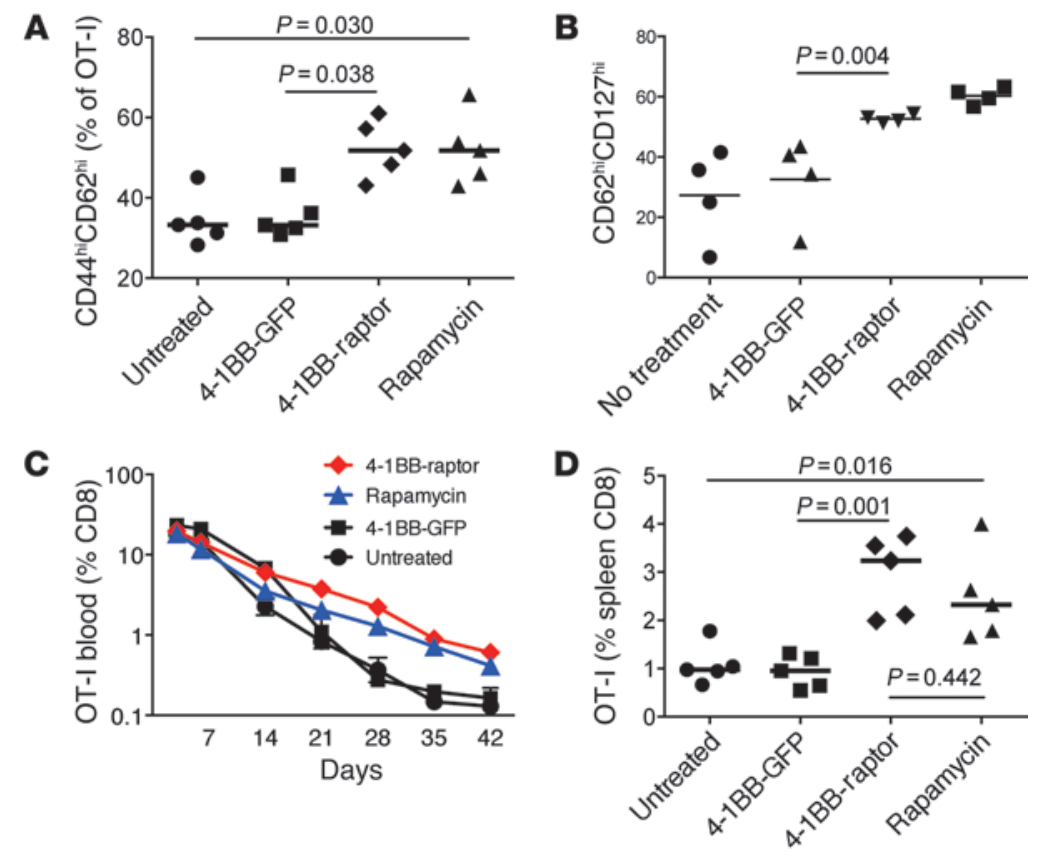

\section{Figure 3}

4-1BB-raptor conjugate promotes the persistence of OT-I cells in mice. CD45.2 C57BL/6 mice were transferred with congenic CD45.1 OT-I cells, activated in situ with OVA peptide, and treated either with rapamycin or with 4-1BB-GFP or 4-1BB-raptor conjugates. Cells were isolated from the blood or spleen, stained with antibodies, and analyzed by flow cytometry. (A) Percentage of CD44h/CD62Li OT-I cells in the spleen on day 5 . Cells were stained with Pacific Blue CD8, PE-CD44, and FITCCD45.1 APC-CD62L antibodies $(n=8)$. (B) Percentage of CD62hi/CD127 hi OT-I cells in the spleen on day 8. Cells were stained with Pacific Blue CD8, APC-CD127, and FITC-CD45.1 PE-CD62L antibodies $(n=2)$. (C) CD8 ${ }^{+}$CD 45.1+ OT-I cells in the blood (5 mice per group). Statistical analysis for 4-1BB raptor versus 4-1BB-GFP, $P=0.0004$; rapamycin versus untreated, $P=0.0003(n=2)$. (D) CD8 ${ }^{+}$CD $45.1^{+}$OT-I cells in the spleen on day $28(n=7)$. the high efficiency (Figure 2, A and E) and specificity (Figure 2, B and D, Supplemental Figure 1A, and Supplemental Figure 2) of aptamer-targeted siRNA delivery to circulating 4-1BB-expressing $\mathrm{CD}^{+} \mathrm{T}$ cells in vivo.

Inhibition of raptor in $C D 8^{+} T$ cells leads to enhanced memory. We next determined whether the 4-1BB-raptor conjugate-mediated mTOR inhibition leads to the generation of enhanced memory $\mathrm{CD}^{+} \mathrm{T}$ cell responses in mice. Figure 3, A and B, show that on days 5 or 8 , a higher proportion of OT-I cells in mice treated with rapamycin or 4-1BB-raptor, but not 4-1BB-GFP, conjugate exhibited a CD62 Li $\mathrm{CD} 44^{\text {hi }}$ or $\mathrm{CD} 62 \mathrm{~L}^{\text {hi }} \mathrm{CD} 127^{\text {hi }}$ phenotype, respectively, indicative of an enhanced proliferative capacity and the potential to develop into memory cells. At later time points, we found increased numbers of OT-I cells in the blood of both rapamycin- and 4-1BB-raptortreated mice (Figure 3C) and on day 28 in the spleen (Figure 3D). Thus, both rapamycin and 4-1BB-raptor treatment enhanced the persistence of the antigen-activated OT-I cells that were enriched for cells with a memory precursor phenotype. Of note, in this and subsequent experiments, mice were injected twice daily with low-dose rapamycin because eight or more daily injections as well as higher doses were detrimental (data not shown). This is different from what was seen in poxvirus (24) or LCMV infection (14) models, perhaps reflecting the differences in the conditions prevailing during priming of the $\mathrm{CD} 8^{+} \mathrm{T}$ cell responses; in LCMV-infected mice or in mice vaccinated with an OVA and costimulatory ligand-expressing poxvirus vector, the $\mathrm{CD} 8^{+} \mathrm{T}$ cells are primed over an extended period of 7 to 35 days under strong inflammatory conditions, whereas in the OVA peptide plus LPS-vaccinated OT-I-bearing mice, the duration and intensity of inflammatory conditions are limited.

We determined the functional status of the persisting OT-I cells by measuring their proliferative capacity and cytotoxic effector functions following antigenic reexposure. Figure 4A shows that in the rapamycin and 4-1BB-raptor, but not 4-1BB-GFP, conjugate-treated mice that were revaccinated with OVA peptide 35 days after priming, the OT-I cells accumulated at higher numbers in the spleen. To assess the cytotoxic effector functions of the OT-I cells, we used an in vivo cytotoxicity assay whereby OT-I cells isolated 30 days after vaccination with OVA peptide were mixed with CFSE ${ }^{\text {hi }}$ OVA-presenting splenocytes and CFSElo ${ }^{\text {lo }}$ control splenocytes, and equal numbers were injected into the recipient mice. The specific lysis of the OVA targets was determined 17 hours later. As shown in Figure 4B, on a per-cell basis, OT-I cells derived from rapamycin-treated mice were less effective than OT-I cells derived from any of the other groups in killing their targets, showing that the rapamycin-generated memory OT-I cells exhibited reduced cytotoxic effector functions.

In separate experiments, we determined the in vitro cytolytic activity of memory OT-I cells isolated from mice treated earlier with either rapamycin or with 4-1BB-raptor or 4-1BB-GFP conjugates using a ${ }^{51} \mathrm{chromium}\left({ }^{51} \mathrm{Cr}\right)$ release assay. As shown in Figure $4 \mathrm{C}$, when unfractionated splenocytes were used as effectors, the killing activity of memory OT-I cells isolated from rapamycin, but not from 4-1BB-raptor or 4-1BB-GFP conjugate-treated mice, was significantly reduced (4-1BB-raptor versus rapamycin, $P=0.028$ ). However, when purified $\mathrm{CD}^{+} \mathrm{T}$ cells were used as effectors, the killing activity of the rapamycin-generated memory OT-I cells was not compromised (4-1BB-raptor versus rapamycin, $P=0.875$ ). While consistent with the in vivo cytotoxicity experiments shown in Figure 4B, the in vitro cytotoxicity experiments suggest that the rapamycin-imprinted cytotoxicity defect is not cell autonomous, but rather is mediated by soluble factors such as IL-10 or TGF- $\beta$, and/or by cells such as Foxp $3^{+}$Tregs or DCs. This is also consistent with the fact that we did not see differences in the expression of the cytotoxic effector molecules perforin, granzyme B, or CD107a in the OT-I cells derived from the rapamycin-treated mice or from mice in any of the other groups (data not shown). Overall, these observations suggest that while both rapamycin and 4-1BB conjugate treatment during priming can promote the development of memory OT-I cells capable of proliferating in response to antigenic stimulation, rapamycin treatment prevented the full acquisition of cytotoxic effector functions by the memory OT-I cells during subsequent antigenic exposure. 
A

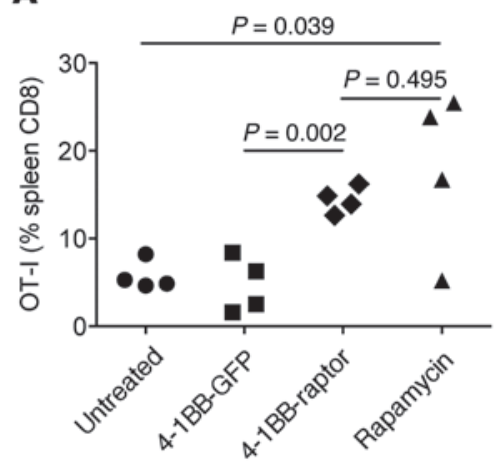

C

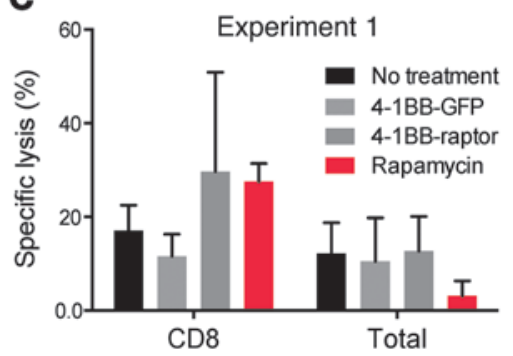

B

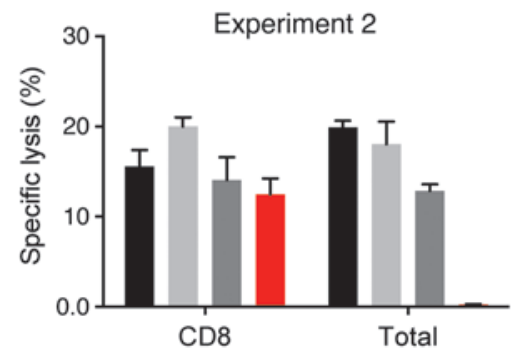

Figure 4

4-1BB-raptor-generated memory OT-I cells exhibit proliferative and cytotoxic effector functions. CD45.2 C57BL/6 mice were transferred with CD45.1 OT-I cells, activated with OVA peptide, and treated either with rapamycin or with 4-1BB-GFP or 4-1BB-raptor conjugates. (A) On day 30 , mice were revaccinated with OVA peptide, and 3 days later the number of $C D 8+C D 45.1$ OT-I cells in the spleen was determined $(n=6)$. (B) On day 30 , splenocytes were isolated,

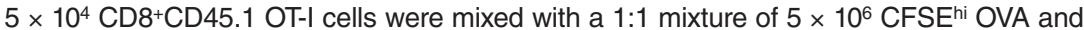
CFSElo influenza NP peptide-pulsed splenocytes, then injected into naive mice. Lysis of OVA targets was determined, calculated as the percentage of reduced CFSEhi cells relative to CFSE $^{\circ}$ cells $(n=8)$. (C) OT-I-bearing mice were vaccinated with OVA peptide and LPS and treated with rapamycin or administered 4-1BB-GFP or 4-1BB-raptor conjugates, as described in Methods. Fourteen days after peptide administration, mice were injected again with peptide and LPS and sacrificed 72 hours later, and splenocytes were isolated (Experiment 1 ), or mice were sacrificed after 30 days, but not injected again with peptide and LPS (Experiment 2). Either total splenocytes or purified CD8 cells containing $10^{4}$ OT-I cells were used as effectors against ${ }^{51} \mathrm{Cr}$-labeled OVA peptide-loaded EL4 targets at a 1:10 effector/target ratio, and specific lysis was determined as described in Methods. Statistical analysis for 4-1BB-raptor versus rapamycin; total splenocytes, $P=0.028$; purified CD8 cells, $P=0.785(n=2)$.

The OT-I CD8 ${ }^{+} \mathrm{T}$ cells that recognize a foreign epitope derived from OVA are a poor model for endogenous tumor antigens, most of which correspond to nonmutated products that elicit low-affinity $\mathrm{T}$ cells. We therefore sought to determine whether the 4-1BB-raptor conjugate is also capable of expanding memory responses directed at low-affinity epitopes encoded in nonmutated endogenous tumor antigens. To this end, we used transgenic Pmel-1 $\mathrm{CD}^{+} \mathrm{T}$ cells that recognize a determinant in the nonmutated gp100 tumor antigen expressed by B16 melanoma tumor cells (48). Pmel-1 cells bind the cognate epitope with much lower affinity than do OT-I cells (Nicholas Restifo, personal communication). When we revaccinated mice 30 days after vaccination with cognate peptide and LPS, the Pmel-1 cells expanded to higher numbers in mice treated with either 4-1BB-raptor conjugate or rapamycin compared with those observed in untreated mice or mice treated with 4-1BB-GFP conjugate (Figure 5A). Overall, reflecting their lower avidity, memory Pmel- 1 cells expanded less effectively than OT-I cells (compare Figure 4A with Figure 5A).
To assess the antitumor effector functions of the memory Pmel- 1 cells, 30 days after vaccination mice were challenged s.c. with B16/F10 tumor cells, and tumor growth was monitored. As shown in Figure 5B, survival was significantly enhanced in mice treated with 4-1BB-raptor conjugate, but not with rapamycin or with 4-1BB-GFP conjugate, despite the fact that rapamycin treatment also enhanced the generation of Pmel- 1 cells, as shown in Figure 5A. We found that the memory Pmel- 1 cells present 32 days after peptide vaccination and rapamycin treatment also exhibited reduced cytotoxic effector functions, as was seen in OT-I-transferred mice. However, the small number of Pmel-1 cells that accumulated in the spleen of the mouse after 32 days, which was about 100-fold less than that of the OT-I cells, precluded a firm conclusion (data not shown).

Aptamer-targeted inhibition of raptor enhancement of vaccine-induced antitumor immunity. Transgenic $\mathrm{CD}^{+} \mathrm{T}$ cells like OT-I and Pmel- 1 provide a useful tool to monitor the immunological parameters of memory development and function and have been all but exclusively used to show that pharmacological inhibition of intracellular mediators can enhance immunological memory and protective immunity $(14,19,22,24,25)$. To assess the therapeutic potential of 4-1BB-raptor conjugates, we tested whether 4-1BB-raptor conjugates can enhance vaccine-induced memory responses against tumor-expressed endogenous antigens that are recognized by the resident, mostly naive $\mathrm{T}$ cell repertoire of the mouse. We first used a prophylactic experimental design whereby mice were vaccinated with GM-CSF-expressing irradiated B16 melanoma cells (GVAX) (49) and then challenged s.c. with parental B16 tumor cells. While a number of tumor antigens expressed in B16 tumor cells have been characterized, the antigens dominating the antitumor response induced by GVAX are not known. It is conceivable that the dominant antigens in the GVAX vaccine correspond to tumor-specific mutated products or to oncofetal antigens, and not to products like TRP2 or gp100 expressed in normal melanocytes. As shown in Figure 6A, treatment with 4-1BB-raptor, but not 4-1BB-GFP or rapamycin, enhanced GVAX-induced antitumor immunity, underscoring the failure of rapamycin treatment to promote memory responses exhibiting antitumor effector functions (Figure 5B). Our antibody depletion experiments suggest that protective immunity elicited by the 4-1BB-raptor conjugate-generated memory response was mediated in part by $\mathrm{CD} 8^{+} \mathrm{T}$ cells (Supplemental Figure 3, $P=0.0052$ ), whereas the contribution of $\mathrm{CD} 4^{+} \mathrm{T}$ cells was not clear, though probable (Supplemental Figure 3, $P=0.064$ ). Given the documented role of $\mathrm{CD}^{+} \mathrm{T}$ cell help in the recall response of memory $\mathrm{CD}^{+} \mathrm{T}$ cells $(10,13,50)$, future studies will be required to examine in depth the contribution of both adaptive and innate arms of the immune response in the 4-1BB-raptor conjugate-mediated enhancement of antitumor immunity. 
A

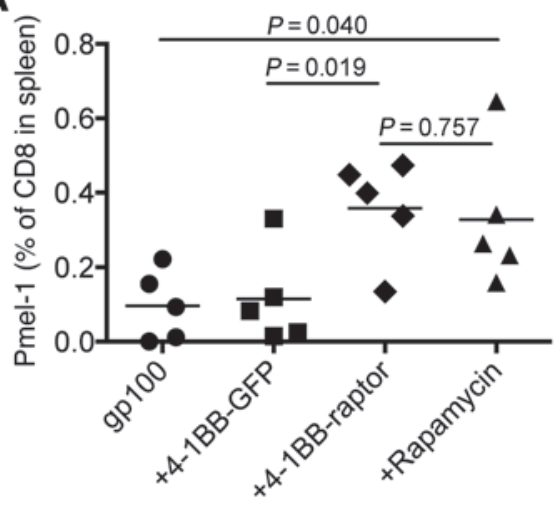

\section{B}

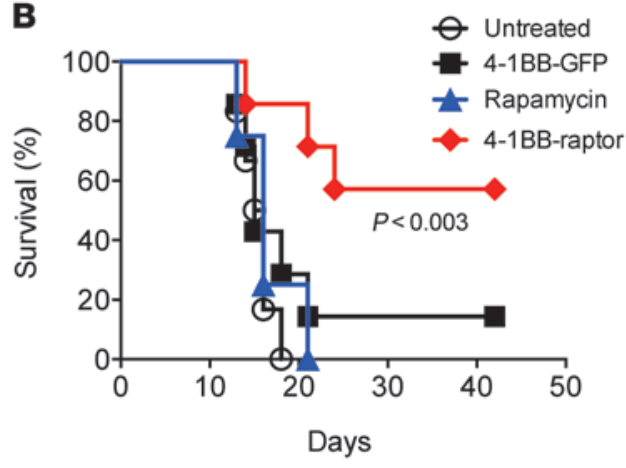

\section{Figure 5}

4-1BB-raptor-generated memory Pmel-1 cells proliferate in response to antigenic challenge and control B16 melanoma tumor growth. CD90.2+ C57BL/6 mice were transferred with congenic CD90.1+ Pmel-1 cells, activated with gp100 peptide 2 days later, and treated either with rapamycin or with 4-1BB-GFP or 4-1BB-raptor conjugates. (A) On day 30, mice were revaccinated with gp100 peptide, and the proportion of CD8 ${ }^{+} \mathrm{CD} 90.1^{+}$ Pmel-1 cells in the spleen was determined by flow cytometry $(n=2)$. (B) On day 30 (day 0 on the $x$ axis), mice were challenged s.c. with $10^{5}$ B16. F10 melanoma cells, and the survival (time to sacrifice when tumors reached $12 \mathrm{~mm}$ in diameter) was determined $(5 \mathrm{mice}$ per group) $(n=3)$.

Given the nontargeted nature of rapamycin administration and its documented immunosuppressive effects mediated by tolerizing DCs $(27,28)$ and promoting Treg differentiation (26), we tested whether the failure to elicit protective immunity shown in Figure 5B and Figure 6A correlated with reduced DC function and/or enhanced Treg accumulation in the rapamycin-treated mice. As shown in Figure 6B, the allogeneic mixed lymphocyte reaction (alloMLR) activity of splenic DCs isolated from the rapamycin-treated mice was reduced compared with that of untreated mice, especially in the setting of GVAX vaccination. In contrast, treatment with 4-1BB-raptor conjugates with or without GVAX vaccination had no adverse effect on DC function. Why GVAX treatment exacerbated DC dysfunction remains to be determined. On the other hand, we saw no measurable differences in the number and Foxp3 expression of Tregs between the rapamycin-treated mice and mice from any other group shown in Figure 6A (data not shown).

To further test the therapeutic potential of enhancing vaccine-induced memory responses, we used therapeutic models in which mice were first implanted with tumor and subsequently subjected to vaccination and treatment with 4-1BB-raptor or 4-1BB-GFP conjugates. To simulate scenarios that mimic clinical settings, experimental conditions were chosen in which vaccination or treatment with aptamer-siRNA conjugates had limited or no antitumor effect. As shown in Figure 6C, 4-1BB-raptor, but not 4-1BB-GFP, conjugates enhanced GVAX-induced antitumor immunity in mice implanted with B16 tumor cells 5 days prior to vaccination and conjugate administration. In this stringent experimental system, neither GVAX vaccination nor treatment with 4-1BB-raptor conjugates alone affected tumor growth. Similarly, 4-1BB-raptor, but not 4-1BB-GFP, conjugates enhanced vaccine-induced protective immunity in mice implanted with 4T1 breast carcinoma tumor cells 7 days before vaccination and conjugate administration (Figure 6D). Vaccination alone or treatment with 4-1BB-GFP or 4-1BB-raptor conjugates had a small antitumor effect that did not reach statistical significance. Since rapamycin was used in our study for comparison purposes only, it was not included in these experiments (Figure 6, C and D) because we could not distinguish between its direct antitumor cytotoxic effects and its enhanced $\mathrm{CD}^{+} \mathrm{T}$ cell memory formation. Indeed, when administered to tumor-bearing mice, rapamycin enhances a protective immunity $(51,52)$ that in one study, using the OT-I model, was directly linked to the generation of enhanced $\mathrm{CD}^{+} \mathrm{T}$ cell memory responses, notably when used at a high, but not low, dose (24).

\section{Discussion}

In this study, we show that aptamer-targeted siRNA-mediated inhibition of mTORC1 signaling in circulating $\mathrm{CD}^{+} \mathrm{T}$ cells leads to the generation of a potent $\mathrm{CD} 8^{+} \mathrm{T}$ cell memory response and enhances vaccine-induced protective immunity. Recent studies using transgenic models and/or targeting the model OVA antigen $(14,19,22,25)$ have provided proof of concept that potentiating memory with pharmacological agents can engender protective immunity against infectious diseases and cancer. Here, we demonstrate the therapeutic potential of promoting immunological memory using nontransgenic experimental models in which mice were implanted with poorly immunogenic tumor

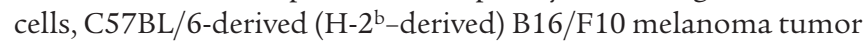
cells (53), and BALB/c-derived ( $\mathrm{H}-2^{\mathrm{d}}$-derived) $4 \mathrm{~T} 1$ breast carcinoma $(54,55)$ tumor cells and then vaccinated with syngeneic irradiated tumor cells presenting endogenous tumor antigens to prime cognate $\mathrm{T}$ cells present in the repertoire of the mouse. We show that in both prophylactic (Figure 6A) and therapeutic (Figure 6, C and D) settings, the 4-1BB aptamer-raptor siRNA conjugate potentiated vaccine-induced protective immunity. These findings underscore the importance of memory responses in protective immunity and show that the approach used in this study could be therapeutically useful.

In this study, we also tested the hypothesis that aptamer-targeted siRNA inhibition of intracellular mediators in vaccine-induced $\mathrm{CD}^{+} \mathrm{T}$ cells will be superior to that of pharmacological agents in terms of enhancing memory $\mathrm{CD}^{+} \mathrm{T}$ cell development and protective antitumor immunity in mice. Cell targeting of RNAi offers important advantages compared with the nontargeted administration of pharmacological agents. Reflecting the broad distribution of their targets, pharmacological agents like rapamycin often 
A
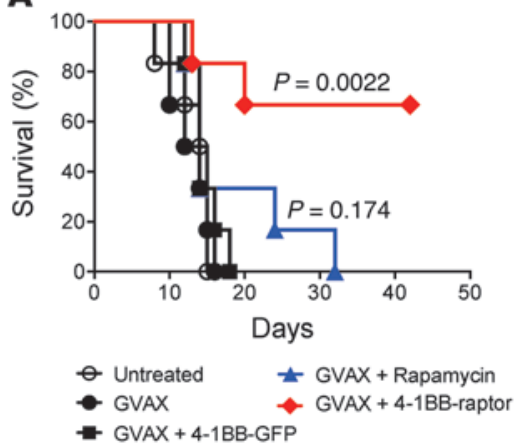

- GVAX + 4-1BB-GFP

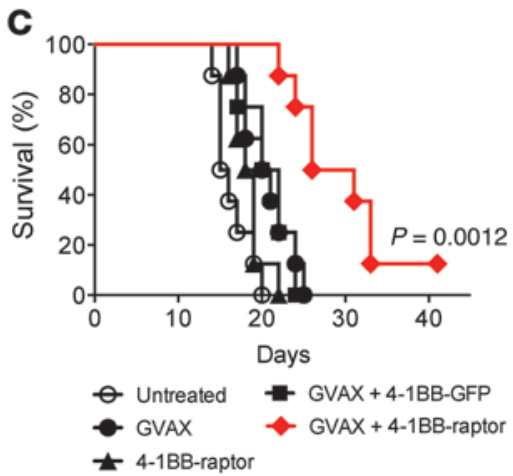

B
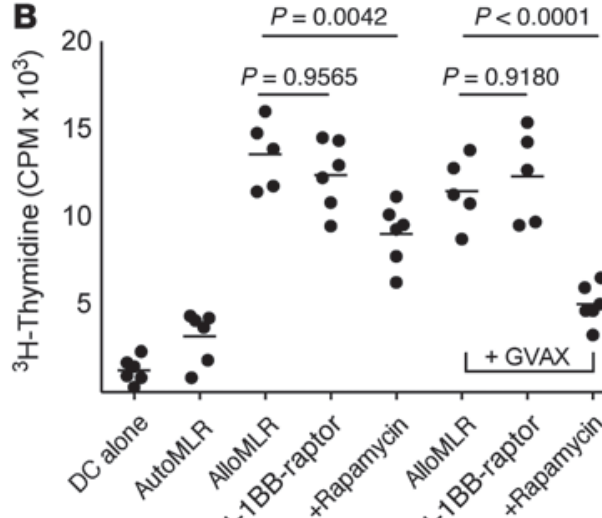

L GVAX

D

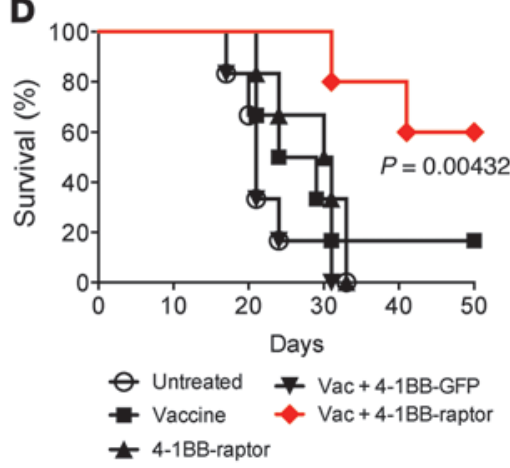

Figure 6

4-1BB-raptor conjugate treatment enhances vaccine-induced protective antitumor immunity. (A) C57BL/6 mice were vaccinated with GVAX and treated either with rapamycin or with 4-1BB-GFP or 4-1BB-raptor conjugates. On day 50 (day 0 on the $x$ axis), mice were challenged with $10^{6}$ B16/F10 melanoma cells, and survival was determined (6 mice per group) $(n=2)$. (B) C57BL/6 mice were injected with PBS or GVAX and treated either with rapamycin or with 4-1BB-GFP or 4-1BB-raptor conjugates. Twenty-four hours after the last treatment, DC-enriched CD $11 \mathrm{c}^{+}$cells were isolated from the spleen, and $10^{4}$ cells were incubated with $4 \times 10^{5} \mathrm{BALB} / \mathrm{c}$ splenocytes. After 4 days, ${ }^{3} \mathrm{H}$-thymidine was added to the culture, and 18 hours later ${ }^{3} \mathrm{H}$-thymidine incorporation was measured $(n=2)$. AutoMLR, autologous mixed lymphocyte reaction. (C) Mice were implanted with B16.F10 tumor cells, vaccinated with GVAX on days 5,8 , and 11 , and/or treated with 4-1BB-GFP or 4-1BB-raptor conjugates on days 6,9 , and 12 (8 mice per group) $(n=6)$. (D) BALB/c mice were implanted with $4 \mathrm{~T} 1$ tumor cells, vaccinated with irradiated B7-1 and MHC class II-expressing 4T1 cells on days 7 , 10 , and 13, and/or treated with 4-1BB-GFP or 4-1BB-raptor conjugates on days 8,11 , and 14 (8 mice per group) $(n=2)$. exhibit pleiotropic effects that can have undesirable consequences such as promoting the development of immunosuppressive Foxp ${ }^{+}$ Tregs and tolerogenic DCs $(30,31)$. Rapamycin can also inhibit both mTORC1 and mTORC2 $(44,45)$, as is also shown in Figure 2B. Given the roles of mTORC2 in cell survival, cytoskeletal organization, and glucose homeostasis $(56,57)$, systemic inhibition of mTORC 2 could be harmful, especially in predisposed individuals.

The underlying premise of this study was that targeted inhibition of mTORC1 in $\mathrm{CD}^{+} \mathrm{T}$ cells using aptamer-targeted RNAi is superior to across-the-board mTOR inhibition with rapamycin in memory formation and protective antitumor immunity. We show here that while both rapamycin and the 4-1BB aptamer-raptor siRNA conjugate elicited a comparable memory response in terms of numbers (Figure 3, B and C) and proliferative capacity (Figure $4 \mathrm{~A}$ and Figure 5A), the rapamycin-generated memory CD8 ${ }^{+}$ $\mathrm{T}$ cells exhibited reduced cytotoxic effector functions (Figure 4, $\mathrm{B}$ and $\mathrm{C})$, DCs isolated from the rapamycin-treated mice exhibited reduced MLR activity (Figure 6B), and the rapamycin-treated mice failed to control tumor growth (Figure $5 \mathrm{~B}$ and Figure $6 \mathrm{~A}$ ). In contrast, we found that systemic administration of 4-1BB aptamer-raptor siRNA conjugates, which downregulated mTORC1, but not $\mathrm{mTORC} 2$, in $\mathrm{CD}^{+} \mathrm{T}$ cells while sparing host cells (Figure 2, A and B), did not adversely affect the cytotoxic effector function of the developing memory cells (Figure 4, B and C) or the alloMLR activity of resident DCs (Figure 6B) and controlled tumor growth (Figure 5B and Figure 6A).

How rapamycin imprints a cytotoxic defect in the developing memory $\mathrm{CD}^{+} \mathrm{T}$ cells remains to be determined. Our observation that the memory OT-I cells exhibited reduced cytolytic activity in vitro when total splenocytes, but not purified $\mathrm{CD}^{+} \mathrm{T}$ cells, were used (Figure 4C) suggests that the cytotoxic defect is not cell intrinsic, but rather is mediated in trans by either cellular subsets such as Tregs or DCs and/or soluble factors such as IL-10 or TGF- $\beta$. The failure to find significant alterations in the level of cytotoxic effector molecules including CD107a, perforin, or granzyme B in the rapamycin-generated memory OT-I cells (data not shown) and the reduced alloMLR activity of resident DCs isolated from rapamycin-treated mice (Figure 6B) are consistent with this possibility. Though our initial examination has not uncovered differences in the Treg population (data not shown), one possibility is that the rapamycin-compromised DCs drive the differentiation of (tumor) antigen-specific Tregs that compromise the cytolytic functions of the reactivated memory $\mathrm{CD}^{+} \mathrm{T}$ cells. Notwithstanding, given the inherent limitations of in vivo cytotoxicity and alloMLR assays, the biological significance of the differences measured in such experimental systems using arbitrarily set experimental conditions is not clear. Future studies will be required to determined whether the reduced cytotoxicity of the memory cells (Figure 4, B and C) and/or the reduced MLR activity of the DCs (Figure 6B) was responsible for the lack of protective immunity in the rapamycin-treated mice (Figure 5B and Figure 6A).

A second advantage of cell targeting is that it reduces the effective dose of siRNA needed to elicit the desired biological effect, thereby reducing the risk of nonspecific immune activation (58) and enhancing the cost-effectiveness of the therapy. Underscoring the efficiency of aptamer-targeted siRNA delivery, we found that two consecutive injections of 1 nmole of 4-BB-raptor conjugate downregulated $\mathrm{mTORC} 1$ activity in over $60 \%$ of the circulating antigen-activated $\mathrm{CD}^{+} \mathrm{T}$ cells (Figure 2A), resulting in an enhanced memory development that was not less effective than that seen 
with rapamycin (Figures 3-5), while three injections of 0.25 nmoles engendered protective antitumor immunity in both prophylactic and therapeutic settings (Figure 6). This compares favorably with nontargeted polymer-formulated siRNA delivery requiring 10 - to 50 -fold higher doses of siRNA to inhibit tumor growth $(59,60)$.

In this study, the raptor siRNA was targeted to antigen-activated $\mathrm{CD}^{+} \mathrm{T}$ cells, peptide-stimulated transgenic OT-I or Pmel- 1 cells, or GVAX vaccine-stimulated tumor-specific $T$ cells using an agonistic dimeric 4-1BB-binding aptamer (39). Thus, the 4-1BB aptamer might have served a dual role: (a) to target the siRNA to 4-1BBexpressing $\mathrm{CD}^{+} \mathrm{T}$ cells, and (b) to promote their survival and potential for differentiating into memory cells. Nonetheless, the agonistic 4-1BB aptamer did not exert a measurable contribution to memory differentiation since 4-1BB aptamer-GFP siRNA conjugates used in every experiment did not affect any of the memory parameters tested. While 4-1BB expression is highly restricted, it is also upregulated on activated conventional CD $4^{+} \mathrm{T}$ cells, Foxp $3^{+}$ Tregs, activated NK cells, CD40-stimulated mature DCs (reviewed in ref. 46), and on proliferating ECs (47). Given that 4-1BB signaling $(46,47)$ and mTOR inhibition $(30,31)$ can exert immune-potentiating or immunosuppressive effects, the positive and negative contributions of 4-1BB-targeted mTOR inhibition in other cells to the protective antitumor immune responses shown in Figure 5B and Figure 6, A, C, and D remain to be determined. It is, however, clear that on balance, targeting raptor siRNA to 4-1BB-expressing cells in vivo enhanced $\mathrm{CD}^{+} \mathrm{T}$ cell memory and protective antitumor immunity (Figures 2-6). Arguably, targeting receptors expressed more exclusively on activated $\mathrm{CD} 8^{+} \mathrm{T}$ cells will further enhance the specificity and therapeutic potential of this approach.

\section{Methods}

Five- to 6-week-old female C57BL/6 (H-2b), BALB/c (H-2d) mice, and transgenic OT-I (42) (H-2b) and Pmel-1 (48) (H-2d) mice were purchased from The Jackson Laboratory and used within 1 to 3 weeks.

\section{Design and characterization of aptamer-siRNA conjugates}

A bivalent murine 4-1BB-binding aptamer (39) was transcribed in vitro using the Durascribe T7 transcription kit (Illumina). The DNA template gggggaattctaatacgactcactataGGGCGGGAGAGAGGAAGA GGGATGGGCGACCGAACGTGCCСTTCAAAGCCGTTCACT AACCAGTGGCATAACCCAGAGGTCGATAGTACTGGATCC CGCCСТССТGCGGCCGAGAGAGGAAGAGGGATGGGCGACCGAACGTGCCCTTCAAAGCCGTTCACTAACCAGTGGCATAACCCAGAGGTCGATAGTACTGGATCGGCCGCTCCCCTCGGGATCTCTTCCAAAA encoded a T7 promoter (lower case) and two aptamers (bold) separated by a single-stranded linker (italicized) fused with the passenger strand of an siRNA (underlined) against raptor mRNA. Raptor mRNA targeting siRNA was characterized as described previously (61). Briefly, candidate sequences were predicted using HPC Dispatcher (City of Hope, Biomedical Informatics Core, Duarte, California, USA), siRNA scales (Department of Human Genetics, University of Utah, Salt Lake City, Utah, USA), and siDESIGN (Dharmacon, Thermo Fisher Scientific) software. Overlapping predictions featuring a low melting temperature $(\mathrm{Tm})$ were selected and screened for specific activity as 4-1BB aptamer conjugates using siCheck assay in transiently transfected HEK293T cells. The aptamer passenger strand fusion transcript was purified by polyacrylamide gel electrophoresis and annealed to a chemically synthesized guide stand. The siCheck assay was used to confirm that target knockdown by the aptamer-siRNA conjugate was comparable to that of free duplex siRNA.

\section{Treatment of $O T$-I cells in vitro with aptamer-siRNA conjugates}

Splenocytes were isolated from CD $45.1^{+}$OT-I mice, and rbcs were lysed with ammonium-chloride-potassium (ACK) solution (Gibco, Life Technologies). Mononuclear cells were washed and incubated overnight with $1 \mathrm{nM}$ of the chicken OVA MHC class I SIINFEKL peptide (AnaSpec) at a density of $10^{6}$ cells per milliliter in complete RPMI-1640 media supplemented with $10 \%$ FBS, essential and nonessential amino acids, Na pyruvate (Gibco, Life Technologies), and $2 \mathrm{ng} / \mathrm{ml}$ murine IL-12 (R\&D Systems). After overnight incubation, cells were washed extensively to remove excess peptide and used in the subsequent analysis. For quantitative PCR (qPCR), cells were plated in triplicate onto 96 -well round-bottom plates $\left(10^{5}\right.$ cells/ well) and incubated with $0.8 \mu \mathrm{M}$ aptamer-siRNA conjugates three times every 8 hours. Thirty-six hours after the last treatment, cells were lysed with Buffer RLT, and RNA was isolated using an RNeasy kit (both from QIAGEN). RNA was quantified using an Agilent 2100 Bioanalyzer (Agilent Technologies). cDNA synthesis was performed using the High Capacity cDNA Reverse Transcription kit (Applied Biosystems). cDNA equivalents of $25 \mathrm{ng}$ of mRNA were used per reaction in a TaqMan qPCR assay using the Step One qPCR machine (Applied Biosystems), with primer sets corresponding to the gene of interest or housekeeping products.

Persistence and proliferation. Cells were plated onto 6-well plates $\left(10^{6} \mathrm{cells} / 5\right.$ $\mathrm{ml}$ ) and incubated with $0.8 \mu \mathrm{M}$ aptamer-siRNA conjugates three times every 8 hours or with rapamycin at $0.5 \mu \mathrm{g} / 5 \mathrm{ml}$. Seventy-two hours after plating, cells were washed and replated $\left(10^{6}\right.$ cells $\left./ 5 \mathrm{ml}\right)$ and incubated in complete RPMI-1640 media with the addition of $5 \mathrm{ng} / \mathrm{ml}$ recombinant IL-7 (R\&D Systems). Every 3 to 5 days, cells were collected, and $10^{6}$ cells were plated per well. On day 25 , the cells were harvested, washed, and plated onto 96-well round-bottom plates, with 10 replicates per condition. Five wells for each condition were treated with $0.01 \mathrm{nM}$ SIINFEKL peptide overnight, and five other wells were treated with control SDYEGRLI influenza NP (50-57) peptide. Eighteen hours later, $1 \mu \mathrm{Ci} \mathrm{H}^{3}$-thymidine was added to each well. Six hours later, cells were harvested, and tritium incorporation was measured using a Wallac MicroBeta scintillation counter (PerkinElmer).

\section{Adoptive transfer of OT-I cells into mice and treatment with rapamycin or aptamer-siRNA conjugates}

Splenocytes from CD45.1 OT-I mice were isolated and enriched for $\mathrm{CD}^{+}$ $\mathrm{T}$ cells using Ly- 2 microbeads (Miltenyi Biotec) to greater than $90 \%$ purity confirmed by flow cytometry. $\mathrm{CD}^{+}$cells $\left(0.5 \times 10^{6}\right)$ were transferred into CD45.2 C57Bl/ 6 mice by tail-vein injection. Forty-eight hours after transfer, the animals were injected i.v. with $10 \mu \mathrm{g}$ SIINFEKL peptide, the chicken OVA dominant class I epitope, and $10 \mu \mathrm{g}$ of E. coli LPS (Sigma-Aldrich) in $100 \mu \mathrm{l}$ PBS. Starting 6 hours after peptide administration, the animals received an i.v. injection of 0.25 nmoles of aptamer-siRNA conjugate in $100 \mu \mathrm{l}$ PBS twice daily, or $1.5 \mu \mathrm{g}$ rapamycin administered i.p. in $200 \mu \mathrm{l}$ PBS immediately after peptide administration, also twice daily.

To measure mTORC1 activity, 36 hours following the second aptamersiRNA conjugate or rapamycin injection, splenocytes were isolated, fixed in $4 \%$ PFA for 10 minutes in RT, and rbcs were lysed with a $0.1 \%$ Triton X-100 PBS solution $\left(37^{\circ} \mathrm{C}\right.$ for 20 minutes). An equal volume of ice-cold wash buffer was added, and the suspension was centrifuged ( $300 \mathrm{~g}$ for 6 minutes at $4^{\circ} \mathrm{C}$ ). Cells were dehydrated with $80 \%$ methanol (prechilled to $-20^{\circ} \mathrm{C}$ ) and incubated on ice for 10 minutes. Then, cells were washed with ice-cold PBS, blocked with ice-cold washing buffer $\left(4^{\circ} \mathrm{C}\right.$ for 30 minutes), and incubated with CD45.1 Pacific Blue (BioLegend) and p-S6-Alexa488 (Cell Signaling Technology) mAbs. To measure mTORC1 and mTORC2 activity in the same preparation, splenocytes were incubated with anti-CD8 and anti-CD45.1 mAbs for 15 minutes at $4^{\circ} \mathrm{C}$, washed, and then fixed and permeabilized using the Cytofix/Cytoperm kit (BD Biosciences) according to the manufacturer's instructions. After permeabilization, cells were stained with anti- 
phospho-S235/236-S6/Alexa488 together with anti-phospho-S473-AKT/ Alexa647 mAbs (Cell Signaling Technology) and analyzed by flow cytometry.

\section{$O T-I$ in vivo cytotoxicity assays}

Splenocytes from C57BL/6 mice were incubated with $100 \mathrm{nM}$ of either the OVA SIINFEKL peptide or the SDYEGRLI influenza NP (50-57) peptide MHC class I-restricted (control) peptide (AnaSpec) in serumfree RPMI- 1640 media for 1 hour at $37^{\circ} \mathrm{C}$, washed and incubated with either $5 \mu \mathrm{M}$ or $0.5 \mu \mathrm{M}$ of CFSE solution in PBS and $2 \%$ FBS at room temperature for 30 minutes, and then washed extensively in cold PBS. On day 30, splenocytes were isolated from OT-I-transferred mice treated with rapamycin or with aptamer-siRNA conjugates, and the proportion of OT-I cells was determined in each mouse by CD8 and CD45.1 costaining. CFSE-labeled OVA and control peptide-loaded splenocytes $\left(5 \times 10^{6}\right)$ were mixed with splenocytes containing $5 \times 10^{4}$ OT-I cells and injected i.v. into $\mathrm{C} 57 \mathrm{Bl} / 6$ mice. Seventeen hours after transfer, splenocytes were isolated, and specific lysis of the OVA peptide-loaded splenocytes was measured as the ratio of remaining SIINFEKL-loaded (CFSEhi)/control peptide-loaded $\left(\mathrm{CFSE}^{\mathrm{lo}}\right)$ targets.

\section{In vitro chromium release assay}

OT-I-bearing mice were vaccinated with OVA peptide and LPS and treated with rapamycin or administered either 4-1BB-GFP or 4-1BB-raptor conjugates, as described above. Fourteen days after peptide administration, the mice were injected again with peptide and LPS, sacrificed 72 hours later, and their splenocytes were isolated. EL4 tumor cells $\left(\mathrm{H}-2^{\mathrm{b}}\right)$ were incubated with $1 \mu \mathrm{M}$ OVA peptide (SIINFEKL), washed and incubated with $0.1 \mu \mathrm{Ci}$ of ${ }^{51} \mathrm{Cr}$. Cells were plated onto a U-bottom 96-well plate with $10^{5}$ cells per well. Total splenocytes or purified CD8 cells from OT-I-bearing mice containing $10^{4} \mathrm{OT}$-I cells were added to wells containing target cells, with 6 wells per condition, and incubated at $37^{\circ} \mathrm{C}$. Sixteen hours later, cell supernatant from each well was harvested, and ${ }^{51} \mathrm{Cr}$ release was measured using a gamma counter. Spontaneous release and nonspecific lysis were determined by coincubation of labeled targets with splenocytes from the control mice bearing no OT-I. Maximal release was measured by adding $0.01 \%$ Triton-X 100 to completely lyse all targets.

\section{$M L R$}

Female C57Bl/6 mice (H-2b) were injected s.c. with $10^{6}$ irradiated (60 Gy) GM-CSF-secreting B16 cells (GVAX). Twenty-four hours later, GVAX-immunized or untreated mice were injected twice daily with aptamer-siRNA conjugate or rapamycin, and 24 hours after the second injection $\mathrm{CD} 11 \mathrm{c}^{+}$ DCs were purified from the spleen using CD11c MicroBeads (Miltenyi Biotec) and irradiated (20 Gy) prior to the MLR assay. In parallel, splenocytes were isolated from female BALB/c mice $(\mathrm{H}-2 \mathrm{~d})$ mice, and $4 \times 10^{5}$ cells were mixed with $10^{4} \mathrm{CD} 11 \mathrm{c}$-enriched CD57BL/6-derived splenocytes and plated onto 96-well round-bottom plates in quintuplicate in complete RPMI-1640 media. Four days after plating, $1 \mu \mathrm{Ci}{ }^{3} \mathrm{H}$-thymidine was added to each well, and tritiated thymidine incorporation was measured 6 hours later using a Wallac MicroBeta scintillation counter (PerkinElmer).

\section{Adoptive transfer of Pmel-1 cells}

Splenocytes $\left(10^{6}\right.$ cells) from Pmel-1 transgenic Thy1.1 C57BL/ 6 mice were transferred to congenic CD90.2 (Ty1.2+) C57BL/6 mice by i.v. injection. Forty-eight hours later, $100 \mu \mathrm{g}$ of KVPRNQDWL human gp100 (25-33) peptide (AnaSpec) and $10 \mu$ g of E. coli LPS were injected i.v., and mice were treated with rapamycin or aptamer-siRNA conjugates as described above. Proliferation and phenotypic analysis was performed as described for OT-I cells, except that cells were costained for CD90.1 (Thy 1.1) instead of CD45.1. To determine the antitumor effector functions of the Pmel-1 cells,
30 days after peptide administration mice were challenged with $10^{5} \mathrm{~B} 16 /$ F10 melanoma tumor cells injected s.c., and tumor growth was monitored daily. Mice were sacrificed when their tumor diameter reached $12 \mathrm{~mm}$.

\section{Tumor immunotherapy studies}

B16/F10 melanoma tumor models. $\mathrm{C} 57 \mathrm{Bl} / 6$ mice were immunized with $10^{6}$ irradiated (60 Gy) GVAX, and 24 hours later mice were treated with 0.25 nmoles of aptamer-siRNA conjugates administered i.v. or with $1.5 \mu \mathrm{g}$ of rapamycin administered i.p. three times daily. On day 50, the mice were challenged s.c. with $10^{6} \mathrm{~B} 16 / \mathrm{F} 10$ tumor cells, and tumor growth was monitored daily. The mice were sacrificed when their tumor diameter reached $12 \mathrm{~mm}$. Alternatively, mice were injected s.c. with $10^{5} \mathrm{~B} 16 / \mathrm{F} 10$ cells and immunized 5 days later with GVAX. Twenty-four hours later, mice were treated with 0.25 nmoles of aptamer-siRNA conjugates administered i.v. or with $1.5 \mu \mathrm{g}$ of rapamycin administered i.p., and this was repeated on days 9 and 12 .

$4 T 1$ breast carcinoma model. BALB/c mice were injected s.c. with $1.0 \times 10^{4}$ 4T1 tumor cells and immunized with a mixture of irradiated B7-1 and MHC class II-expressing 4T1 cells (62) and then treated with aptamersiRNA conjugates as described above.

\section{Statistics}

Unpaired, 2-tailed Student's $t$ tests were performed between individual treatment groups using GraphPad Prism, version 5.0 (GraphPad Software). $P$ values less than or equal to 0.05 were considered statistically significant. For survival curves, a log-rank test was performed to determine statistical significance for differences in survival times between treatment groups, with $P$ values below 0.05 being considered statistically significant.

\section{Study approval}

The facilities at the University of Miami Division of Veterinary Resources are fully accredited by the Association for Assessment and Accreditation of Laboratory Animal Care and the USDA. An OLAW assurance is on file, ensuring that humane animal care and use practices, as outlined in the Guide for the Care and Use of Laboratory Animals (NIH publication no. 85-23. Revised 1985) are followed. All mice were maintained according to the guidelines established by the US Department of Agriculture and the American Association for Accreditation of Laboratory Animal Care (AAALAC). This project was approved by the IACUC of the University of Miami School of Medicine.

\section{Acknowledgments}

We thank Randall Brenneman for his assistance in writing the manuscript. Funding was provided by a bequest from the Dodson Estate and the Sylvester Comprehensive Cancer Center (Miller School of Medicine, University of Miami, Miami, Florida, USA) and by a grant (KG090348) from the Susan G. Komen for the Cure of Breast Cancer Foundation. A. Berezhnoy was responsible for designing and testing the aptamer-siRNA conjugates, in vivo memory studies, and tumor immunotherapy experiments. A. Levay was responsible for generating aptamer-siRNA conjugates and murine immunotherapy studies. I. Castro and T.R. Malek were responsible for designing the OT-I experiments and assisted in the writing of the manuscript. E. Gilboa oversaw the project and was responsible for writing the manuscript.

Received for publication March 12, 2013, and accepted in revised form September 19, 2013.

Address correspondence to: Eli Gilboa, University of Miami, Miller School of Medicine, 1550 NW 10th Avenue, Medical Campus, Fox Building 306 (M710), Miami, Florida 33136, USA. Phone: 305.243.1767; Fax: 305.243.4409; E-mail: egilboa@med.miami.edu. 
1. Klebanoff CA, Gattinoni L, Restifo NP. CD8 ${ }^{+}$T-cell memory in tumor immunology and immunotherapy. Immunol Rev. 2006;211:214-224.

2. Letvin NL, et al. Preserved $\mathrm{CD}^{+}$central memory $\mathrm{T}$ cells and survival in vaccinated SIV-challenged monkeys. Science. 2006;312(5779):1530-1533.

3. Mattapallil JJ, et al. Vaccination preserves CD4 memory $\mathrm{T}$ cells during acute simian immunodeficiency virus challenge. J Exp Med. 2006;203(6):1533-1541.

4. Vaccari M, Trindade CJ, Venzon D, Zanetti M, Franchini G. Vaccine-induced $\mathrm{CD}^{+}$central memory $\mathrm{T}$ cells in protection from simian AIDS. J Immunol. 2005;175(6):3502-3507.

5 . Kalos M, et al. T cells with chimeric antigen receptors have potent antitumor effects and can establish memory in patients with advanced leukemia. Sci Transl Med. 2011;3(95):95ra73.

6. Louis CU, et al. Antitumor activity and longterm fate of chimeric antigen receptor-positive $\mathrm{T}$ cells in patients with neuroblastoma. Blood. 2011;118(23):6050-6056.

7. Robbins PF, et al. Cutting edge: persistence of transferred lymphocyte clonotypes correlates with cancer regression in patients receiving cell transfer therapy. J Immunol. 2004;173(12):7125-7130.

8. Zhou J, Shen X, Huang J, Hodes RJ, Rosenberg SA, Robbins PF. Telomere length of transferred lymphocytes correlates with in vivo persistence and tumor regression in melanoma patients receiving cell transfer therapy. J Immunol. 2005;175(10):7046-7052.

9. Rosenberg SA, et al. Durable complete responses in heavily pretreated patients with metastatic melanoma using T-cell transfer immunotherapy. Clin Cancer Res. 2011;17(13):4550-4557.

10. Cui W, Kaech SM. Generation of effector CD8 ${ }^{+}$ $\mathrm{T}$ cells and their conversion to memory $\mathrm{T}$ cells Immunol Rev. 2010;236:151-166.

11. Harty JT, Badovinac VP. Shaping and reshaping $\mathrm{CD}^{+}$T-cell memory. Nat Rev Immunol. 2008; 8(2):107-119.

12. Mescher MF, et al. Signals required for programming effector and memory development by $\mathrm{CD} 8^{+}$ T cells. Immunol Rev. 2006;211:81-92.

13. Williams MA, Bevan MJ. Effector and memory CTL differentiation. Annu Rev Immunol. 2007;25:171-192.

14. Araki K, et al. mTOR regulates memory CD8 T-cell differentiation. Nature. 2009;460(7251):108-112.

15. Joshi NS, et al. Inflammation directs memory precursor and short-lived effector $\mathrm{CD} 8\left(^{+}\right) \mathrm{T}$ cell fates via the graded expression of T-bet transcription factor. Immunity. 2007;27(2):281-295.

16. Kallies A, Xin A, Belz GT, Nutt SL. Blimp-1 transcription factor is required for the differentiation of effector $\mathrm{CD} 8\left(^{+}\right) \mathrm{T}$ cells and memory responses. Immunity. 2009;31(2):283-295.

17. Rutishauser RL, et al. Transcriptional repressor Blimp-1 promotes CD8(+) T cell terminal differentiation and represses the acquisition of central memory T cell properties. Immunity. 2009;31(2):296-308.

18. Crotty S, Johnston RJ, Schoenberger SP. Effectors and memories: Bcl-6 and Blimp-1 in T and B lymphocyte differentiation. Nat Immunol. 2010; 11(2):114-120.

19. Gattinoni L, et al. Wnt signaling arrests effector $\mathrm{T}$ cell differentiation and generates CD8+ memory stem cells. Nat Med. 2009;15(7):808-813.

20. Intlekofer AM, et al. Effector and memory CD8 ${ }^{+}$ $\mathrm{T}$ cell fate coupled by T-bet and eomesodermin. Nat Immunol. 2005;6(12):1236-1244.

21. Zhou X, Yu S, Zhao DM, Harty JT, Badovinac VP, Xue HH. Differentiation and persistence of memory CD8 $\left(^{+}\right) \mathrm{T}$ cells depend on $\mathrm{T}$ cell factor 1 . Immunity. 2010;33(2):229-240.

22. Pearce EL, et al. Enhancing CD8 T-cell memory by modulating fatty acid metabolism. Nature.
2009;460(7251):103-107.

23. Jeannet G, Boudousquie C, Gardiol N, Kang J Huelsken J, Held W. Essential role of the Wnt pathway effector Tcf-1 for the establishment of functional CD8 T cell memory. Proc Natl Acad Sci U S A. 2010;107(21):9777-9782

24. Li Q, et al. Regulating mammalian target of rapamycin to tune vaccination-induced $\mathrm{CD} 8\left(^{+}\right)$ $\mathrm{T}$ cell responses for tumor immunity. J Immunol. 2012;188(7):3080-3087.

25. Rao RR, Li Q, Odunsi K, Shrikant PA. The mTOR kinase determines effector versus memory $\mathrm{CD}^{+}$ $\mathrm{T}$ cell fate by regulating the expression of transcription factors T-bet and Eomesodermin. Immunity. 2010;32(1):67-78.

26. Delgoffe GM, et al. The mTOR kinase differentially regulates effector and regulatory $\mathrm{T}$ cell lineage commitment. Immunity. 2009;30(6):832-844

27. Hackstein H, et al. Rapamycin inhibits IL-4induced dendritic cell maturation in vitro and dendritic cell mobilization and function in vivo. Blood. 2003;101(11):4457-4463

28. Turnquist HR, Raimondi G, Zahorchak AF, Fischer RT, Wang Z, Thomson AW. Rapamycin-conditioned dendritic cells are poor stimulators of allogeneic $\mathrm{CD}^{+} \mathrm{T}$ cells, but enrich for antigen-specific Foxp $3^{+}$ $T$ regulatory cells and promote organ transplant tolerance. J Immunol. 2007;178(11):7018-7031.

29. Sinclair LV, et al. Phosphatidylinositol-3-OH kinase and nutrient-sensing $\mathrm{mTOR}$ pathways control T lymphocyte trafficking. Nat Immunol. 2008;9(5):513-521.

30. Powell JD, Pollizzi KN, Heikamp EB, Horton MR. Regulation of immune responses by mTOR. Annu Rev Immunol. 2012;30:39-68.

31. Thomson AW, Turnquist HR, Raimondi G. Immunoregulatory functions of mTOR inhibition. Nat Rev Immunol. 2009;9(5):324-337.

32. Keefe AD, Pai S, Ellington A. Aptamers as therapeutics. Nat Rev Drug Discov. 2010;9(7):537-550.

33. Dassie JP, et al. Systemic administration of optimized aptamer-siRNA chimeras promotes regression of PSMA-expressing tumors. Nat Biotechnol. 2009;27(9):839-849.

34. Ni X, et al. Prostate-targeted radiosensitization via aptamer-shRNA chimeras in human tumor xenografts. J Clin Invest. 2011;121(6):2383-2390.

35. Neff CP, et al. An aptamer-siRNA chimera suppresses HIV-1 viral loads and protects from helper CD4( $\left(^{+}\right) \mathrm{T}$ cell decline in humanized mice. Sci Transl Med. 2011;3(66):66ra66

36. Wheeler LA, et al. Inhibition of HIV transmission in human cervicovaginal explants and humanized mice using CD4 aptamer-siRNA chimeras. J Clin Invest. 2011;121(6):2401-2412.

37. Pastor F, Kolonias D, Giangrande PH, Gilboa E. Induction of tumor immunity by targeted inhibition of nonsense mediated mRNA decay. Nature. 2010;465(7295):227-231.

38. Pastor F, Kolonias D, McNamara JO, Gilboa E. Targeting 4-1BB costimulation to disseminated tumor lesions with bi-specific oligonucleotide aptamers. Mol Ther. 2011;19(10):1878-1886.

39. McNamara JO, et al. Multivalent 4-1BB binding aptamers costimulate CD8 T cells and inhibit tumor growth in mice. J Clin Invest. 2008;118(1):376-386.

40. Kwon B, Lee HW, Kwon BS. New insights into the role of 4-1BB in immune responses: beyond $\mathrm{CD}^{+}$ $\mathrm{T}$ cells. Trends Immunol. 2002;23(8):378-380.

41. Wang C, Lin GH, McPherson AJ, Watts TH. Immune regulation by 4-1BB and 4-1BBL: complexities and challenges. Immunol Rev. 2009;229(1):192-215.

42. Hogquist KA, Jameson SC, Heath WR, Howard JL, Bevan MJ, Carbone FR. T cell receptor antagonist peptides induce positive selection. Cell. 1994;76(1):17-27. 43. Takemoto N, Intlekofer AM, Northrup JT, Wherry
EJ, Reiner SL. Cutting Edge: IL-12 inversely regulates T-bet and eomesodermin expression during pathogen-induced $\mathrm{CD}^{+} \mathrm{T}$ cell differentiation. Immunol. 2006;177(11):7515-7519.

44. Delgoffe GM, et al. The kinase mTOR regulates the differentiation of helper $\mathrm{T}$ cells through the selective activation of signaling by MTORC1 and mTORC2. Nat Immunol. 2011;12(4):295-303.

45. Sarbassov DD, et al. Prolonged rapamycin treatment inhibits mTORC2 assembly and Akt/PKB. Mol Cell. 2006;22(2):159-168.

46. Vinay DS, Kwon BS. 4-1BB signaling beyond T cells. Cell Mol Immunol. 2011;8(4):281-284.

47. Palazon A, et al. Agonist anti-CD137 mAb act on tumor endothelial cells to enhance recruitment of activated $\mathrm{T}$ lymphocytes. Cancer Res. 2011;71(3):801-811.

48. Overwijk WW, et al. Tumor regression and autoimmunity after reversal of a functionally tolerant state of self-reactive CD8+ T cells. J Exp Med. 2003;198(4):569-580

49. Dranoff $G$, et al. Vaccination with irradiated tumor cells engineered to secrete murine granulocyte-macrophage colony-stimulating factor stimulates potent, specific, and long-lasting anti-tumor immunity. Proc Natl Acad Sci U S A. 1993;90(8):3539-3543.

50. Wiesel M, Oxenius A. From crucial to negligible: functional CD8(+) T-cell responses and their dependence on CD4(+) T-cell help. Eur J Immunol. 2012;42(5):1080-1088.

51. Jiang Q, et al. mTOR kinase inhibitor AZD8055 enhances the immunotherapeutic activity of an agonist CD40 antibody in cancer treatment. Cancer Res. 2011;71(12):4074-4084.

52. Wang Y, Wang XY, Subjeck JR, Shrikant PA, Kim HL. Temsirolimus, an mTOR inhibitor, enhances anti-tumour effects of heat shock protein cancer vaccines. Br J Cancer. 2011;104(4):643-652.

53. Fidler IJ. Biological behavior of malignant melanoma cells correlated to their survival in vivo. Cancer Res. 1975;35(1):218-224.

54. Miller FR, Miller BE, Heppner GH. Characterization of metastatic heterogeneity among subpopulations of a single mouse mammary tumor: heterogeneity in phenotypic stability. Invasion Metastasis. 1983;3(1):22-31.

55. Aslakson CJ, Miller FR. Selective events in the metastatic process defined by analysis of the sequential dissemination of subpopulations of a mouse mammary tumor. Cancer Res. 1992;52(6):1399-1405.

56. Oh WJ, Jacinto E. mTOR complex 2 signaling and functions. Cell Cycle. 2011;10(14):2305-2316.

57. Lamming DW, et al. Rapamycin-induced insulin resistance is mediated by mTORC2 loss and uncoupled from longevity. Science. 2012;335(6076):1638-1643.

58. Judge AD, Sood V, Shaw JR, Fang D, McClintock K, MacLachlan I. Sequence-dependent stimulation of the mammalian innate immune response by synthetic siRNA. Nat Biotechnol. 2005;23(4):457-462.

59. Judge AD, et al. Confirming the RNAi-mediated mechanism of action of siRNA-based cancer therapeutics in mice. J Clin Invest. 2009;119(3):661-673.

60. Ren Y, et al. Targeted Tumor-Penetrating siRNA Nanocomplexes for Credentialing the Ovarian Cancer Oncogene ID4. Sci Transl Med. 2012;4(147):147ra112.

61. Berezhnoy A, Brenneman R, Bajgelman M, Seales D, Gilboa E. Thermal stability of siRNA modulates aptamer-conjugated siRNA inhibition. Mol Ther Nucleic Acids. 2012;1:e51-e59.

62. Baskar S, Glimcher L, Nabavi N, Jones RT, Ostrand-Rosenberg S. Major histocompatibility complex class $\mathrm{II}^{+} \mathrm{B} 7-1^{+}$tumor cells are potent vaccines for stimulating tumor rejection in tumor-bearing mice. J Exp Med. 1995;181(2):619-629. 